\title{
Transient enhancement of photorefractive gratings in lead germanate by homogeneous pyroelectric fields
}

\author{
Xuefeng Yue* and S. Mendricks \\ Fachbereich Physik der Universität Osnabrück, Barbarastrasse 7, D-49069 Osnabrück, Germany \\ T. Nikolajsen \\ Optics and Fluid Department, Risb National Laboratory, DK-400o Roskilde, Denmark \\ H. Hesse, D. Kip, and E. Krätzig \\ Fachbereich Physik der Universität Osnabrück, Barbarastrasse 7, D-49069 Osnabrück, Germany
}

Received August 11, 1998; revised manuscript received November 2, 1998

\begin{abstract}
During holographic recording in pure $\left(\mathrm{Pb}_{5} \mathrm{Ge}_{3} \mathrm{O}_{11}\right)$ and doped $\left[\left(\mathrm{Pb}_{1-x} \mathrm{Ba}_{x}\right)_{5} \mathrm{Ge}_{3} \mathrm{O}_{11}\right]$ lead germanate crystals, the diffraction efficiency is transiently enhanced at the initial stage. The enhancement is studied as a function of writing-beam intensity and of dark delay time between two successive recording processes and with open- or short-circuited crystal surfaces. Homogeneous pyroelectric fields that arise from heating of the crystals by cw illumination are revealed to be the main mechanism for diffraction efficiency enhancement. The combined effect of pyroelectric fields and charge compensation is analyzed and used for the explanation of the observed phenomena. (C) 1999 Optical Society of America [S0740-3224(99)00103-4]
\end{abstract}

OCIS code: 160.5320.

\section{INTRODUCTION}

Great interest in photorefractive crystals has persisted for approximately four decades. Photorefractive crystals offer great promise for applications in optical data processing and storage. $^{1}$ The photorefractive effect in electro-optic crystals consists of several steps: Inhomogeneous illumination excites charge carriers, and transport and redistribution of these charge carriers form space-charge fields, which modulate the refractive index by means of the electro-optic effect. ${ }^{2}$ It is generally considered that the free charge carriers move because of diffusion, drift in the external electric field, and the photovoltaic effect.

In 1978 Vinetskii and Itskovskii ${ }^{3}$ discussed the role of the pyroelectric effect in light-induced charge transfer. They concluded that the pyroelectric field is of importance only in crystals with high absorption and large grating spacing. In 1991 Ducharme $^{4}$ reported that transient phase gratings can arise from pyroelectric and electrooptic effects. The influence of a pyroelectric chargedriving force on the photorefractive effect was demonstrated experimentally in 1993 by Buse. ${ }^{5}$ In these experiments a pulsed laser (wavelength, $\lambda=532 \mathrm{~nm}$; pulse duration, $t_{p}=20 \mathrm{~ns}$ ) was used such that intensities as high as $320 \mathrm{GW} / \mathrm{m}^{2}$ were reached. Homogeneous pyroelectric fields can also enhance the photorefractive effect. In 1990 the observation of enhanced photorefractive beam fanning because of the pyroelectric effect was reported. ${ }^{6}$ Later the transient enhancement of amplification during two-wave mixing in Cu-doped $\mathrm{KN}$ SBN crystal was mentioned and attributed to pyroelectric fields, ${ }^{7}$ but no detailed experimental results and analyses were given. More recently Korneev et al. ${ }^{8}$ observed an enhanced photorefractive grating by heating or cooling and by homogeneous illumination of a strontium barium niobate crystal with a cw laser. In these experiments the standard phase-modulation technique was used. As was already stated in Ref. 8, pyroelectric fields are of importance for applications of photorefractive crystals such as holographic recording and optical switching as well as for the fundamental understanding of photorefractive charge-transport processes in pyroelectric crystals. In this paper we present direct measurements of transient enhancement of diffraction efficiency during holographic recording in pure and doped lead germanate crystals, which recently have been found to show interesting photorefractive effects. ${ }^{9,10}$ A fast grating and a slow grating with substantially different response time constants can be produced during holographic recording. Here we focus on the dynamic behavior of the fast grating. The enhancement of photorefractive gratings is studied as a function of writing-beam intensity and dark delay time between two successive recording processes: Holographic recording is carried out under the conditions that the crystal surfaces are open or short circuited. To analyze the results we consider light-induced temperature 
Table 1. Descriptions of Our Samples and Some of Their Physical Parameters ${ }^{a}$

\begin{tabular}{llccrrr}
\hline Abbreviation & Crystal and Dopant & Dimensions $a \times b \times c\left(\mathrm{~mm}^{3}\right)$ & $\sigma_{d}(\mathrm{~cm} \Omega)^{-1}$ & $\sigma_{p}\left({\mathrm{~cm} \Omega)^{-1}}^{2}\left(\mathrm{~cm}^{-1}\right)\right.$ & $M$ \\
\hline PGO & $\mathrm{Pb}_{5} \mathrm{Ge}_{3} \mathrm{O}_{11}$ & $2.40 \times 6.20 \times 5.50$ & $2.2 \times 10^{-10}$ & $3.3 \times 10^{-9}$ & 3.2 & 1.3 \\
PBGO:0.02 & $\left(\mathrm{Pb}_{0.98} \mathrm{Ba}_{0.02}\right)_{5} \mathrm{Ge}_{3} \mathrm{O}_{11}$ & $2.60 \times 4.10 \times 4.40$ & $2.3 \times 10^{-11}$ & $4.2 \times 10^{-10}$ & 1.8 & 2.4 \\
PBGO:0.04 & $\left(\mathrm{Pb}_{0.96} \mathrm{Ba}_{0.04}\right)_{5} \mathrm{Ge}_{3} \mathrm{O}_{11}$ & $3.10 \times 6.50 \times 6.76$ & $3.8 \times 10^{-12}$ & $2.5 \times 10^{-10}$ & 2.0 & 3.3 \\
\hline
\end{tabular}

${ }^{a}$ Here $\sigma_{d}$ is the dark conductivity, $\sigma_{p}$ is the photoconductivity for an intensity $I_{0}=0.4 \mathrm{~W} / \mathrm{cm}^{2}$ at a wavelength $\lambda=488 \mathrm{~nm}, \alpha$ is the absorption coefficient at a wavelength $\lambda=488 \mathrm{~nm}$, and $M$ is the transient enhancement factor of the photorefractive gratings during recording with an intensity $I_{0}$ $=16 \mathrm{~W} / \mathrm{cm}^{2}$ at a wavelength of $488 \mathrm{~nm}$.

changes, pyroelectric fields, and compensation of the pyroelectric field by photoexcited charges.

\section{SAMPLES AND EXPERIMENTS}

$\mathrm{Pb}_{5} \mathrm{Ge}_{3} \mathrm{O}_{11}$ (PGO) and $\left(\mathrm{Pb}_{1-x} \mathrm{Ba}_{x}\right)_{5} \mathrm{Ge}_{3} \mathrm{O}_{11}$ (PBGO:0.02 and PBGO:0.04; $x=0.02$, 0.04, respectively) crystals were grown by the Czochralski method at the Crystal Growth Laboratory of the University of Osnabrück. They were cut, polished to optical quality, and poled to the single-domain state. A description of the samples used in our experiments and some of their physical parameters are given in Table 1.

A holographic setup is used to measure the dynamic properties of the fast grating in pure and Ba-doped lead germanate crystals: Two expanded beams of an $\mathrm{Ar}^{+}$laser (wavelength, $\lambda=488 \mathrm{~nm}$ ) of the same intensity and with extraordinary polarization are utilized to write gratings with grating vectors parallel to the $c$ axes of the crystals. The grating spacing in all experiments is $1.2 \mu \mathrm{m}$. The formation of the gratings is monitored by a weak extraordinarily polarized He-Ne laser beam $(\lambda=633 \mathrm{~nm})$ incident at the Bragg angle. The diffracted red beam is detected by photodiodes and recorded with a storage oscilloscope.

Here we define two kinds of boundary condition: openand short-circuit recording. The former condition, in which the two $c$ faces of the crystal are not connected, is widely used in holographic recording. In photovoltaic or pyroelectric crystals, open-circuit recording introduces a voltage across the crystal, whereas under short-circuited conditions the voltage remains zero. ${ }^{11}$ Furthermore, two different initial conditions are applied in the present measurements: recording with and without preillumination. One of the writing beams is used as the preillumination beam, and one can record directly by turning on the other writing beam.

\section{EXPERIMENTAL RESULTS}

First we record a grating in sample PBGO:0.02 by using an open circuit. If the beam intensity $I_{0}$ is low, i.e., $I_{0}$ $<1 \mathrm{~W} / \mathrm{cm}^{2}$, the diffracted intensity $I_{d}$ increases to a steady-state value in a short time. But, if we increase the intensity, a transient enhancement of diffraction efficiency at the initial stage of holographic recording is observed. This behavior is shown in Fig. 1 by the solid curve. For an intensity $I_{0}=16 \mathrm{~W} / \mathrm{cm}^{2}$ of the writing beams a transient maximum value of diffracted intensity can be reached that is 2.4 times higher than the steadystate value (curve B of Fig. 1). Then we erase the grating

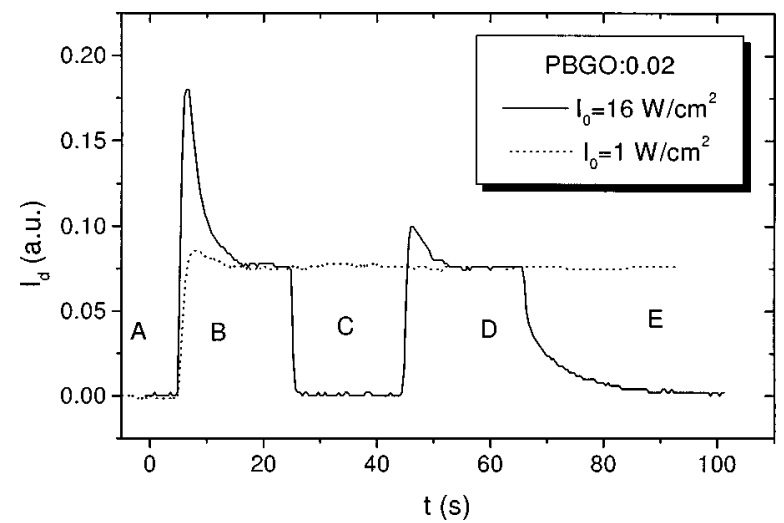

Fig. 1. Evolution of diffracted beam intensity $I_{d}$ during holographic recording in sample PBGO:0.02. The intensities of the writing beams are 16 and $1 \mathrm{~W} / \mathrm{cm}^{2}$. Solid curve: A, dark; B, recording; C, optical erasure; $\mathrm{D}$, recording directly after optical erasure; E, dark decay of the recorded grating. Dotted curve: $\mathrm{A}$, dark; $\mathrm{B}-\mathrm{E}$, one recording process.

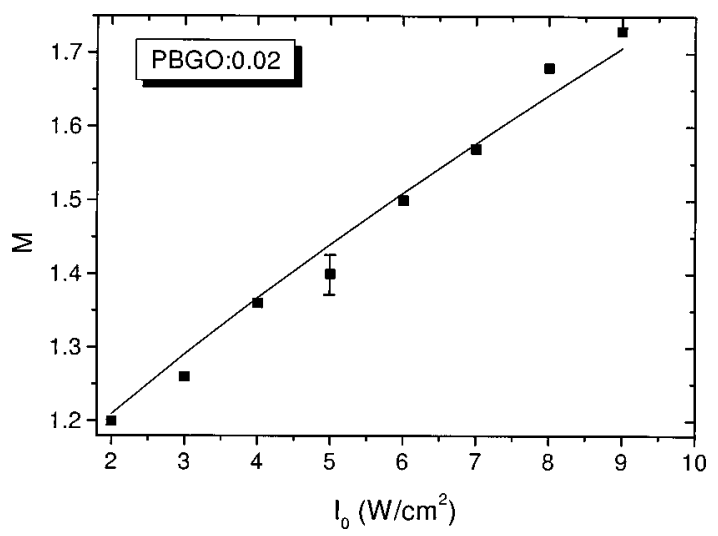

Fig. 2. Enhancement factor $M$ as a function of intensity $I_{0}$ of both writing beams. Here $M$ is defined as the ratio between maximum and steady-state diffracted intensity. The solid curve is a fit according to Eq. (9).

optically by turning off one of the writing beams (curve C). The following recording (curve D) is made with preillumination. Here again a transient enhancement during the new recording is observed, but it is much smaller than that for recording without preillumination. In the last part (curve E) the recorded grating is allowed to decay in the dark.

We define the enhancement factor $M$ as the ratio between the maximum diffracted intensity and the steadystate value. We find that $M$ depends strongly on the writing beam's intensity for recording without preillumi- 
nation. We show the measured dependence in Fig. 2, from which we see a linear increase of enhancement factor $M$ with writing-beam intensity.

In the following experiment we write a grating for 100 s with writing beams of intensity $I_{0}=8 \mathrm{~W} / \mathrm{cm}^{2}$, allow the grating to decay in the dark for different time intervals $t_{d}$, and then start a new recording. After a time $t_{d}$ $=20 \mathrm{~s}$ the grating is already completely decayed. Enhancement factor $M$ for the new recording depends strongly on the dark delay time, as shown in Fig. 3. Factor $M$ increases monotonically with dark delay time and saturates at a value of 1.7. The solid curve in Fig. 3 is a fit based on a theoretical factor that is explained in Section 4.

To achieve short-circuit recording we cover the four lateral faces of the crystal with conducting silver paste. For an intensity of the writing beams of $I_{0}=8 \mathrm{~W} / \mathrm{cm}^{2}$ the evolution of the diffracted beam's intensity during holographic recording and without preillumination is as shown in Fig. 4. For comparison we present in the same figure open-circuit recording at the same intensity. Clearly, for short-circuit recording the transient enhancement $(M \sim 1.2)$ of the diffracted beam is smaller than in the open-circuit case $(M \sim 1.8)$.

We repeat the above experiments in different samples, including PBGO:0.04 and the nominally pure sample PGO. In sample PBGO:0.04 transient enhancement is more pronounced. For a light intensity of $I_{0}$ $=16 \mathrm{~W} / \mathrm{cm}^{2}$ the enhancement factor is $M=3.3$. In sample PGO, however, the enhancement factor is only 1.3 under the same conditions.

Finally we measure the pyroelectric current in sample PGO. Figure 5 shows the time evolution of the pyroelectric current density. The wavelength and the intensity of the illuminating light are $515 \mathrm{~nm}$ and $150 \mathrm{~W} / \mathrm{m}^{2}$, respectively. The current is zero before illumination and shows a maximum at the beginning of illumination, followed by a decrease to a stationary state where the current density is again zero. After illumination is switched off, the opposite effect is observed. The decrease in $|j|$, the absolute value of the current density after it has reached a maxi-

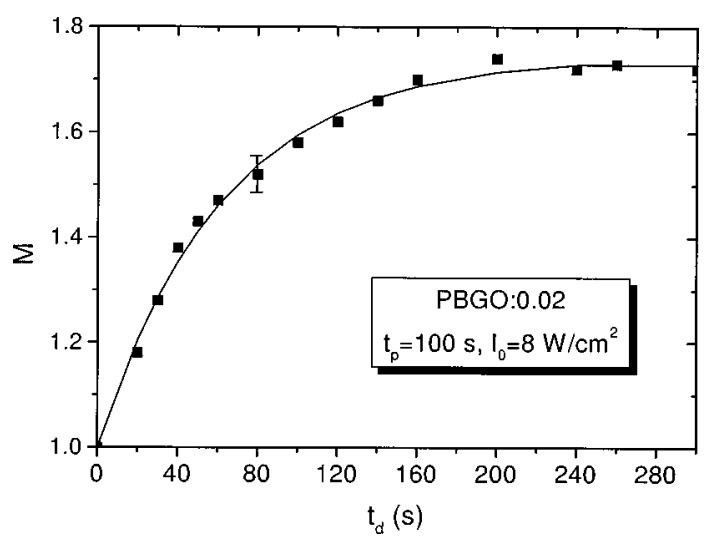

Fig. 3. Enhancement factor $M$ of the photorefractive grating as a function of dark delay time $t_{d}$ in PBGO:0.02. The intensities of the writing beams and the writing time before dark delay are $I_{0}$ and $t_{p}$, respectively. The solid curve is a fit according to Eq. (11).

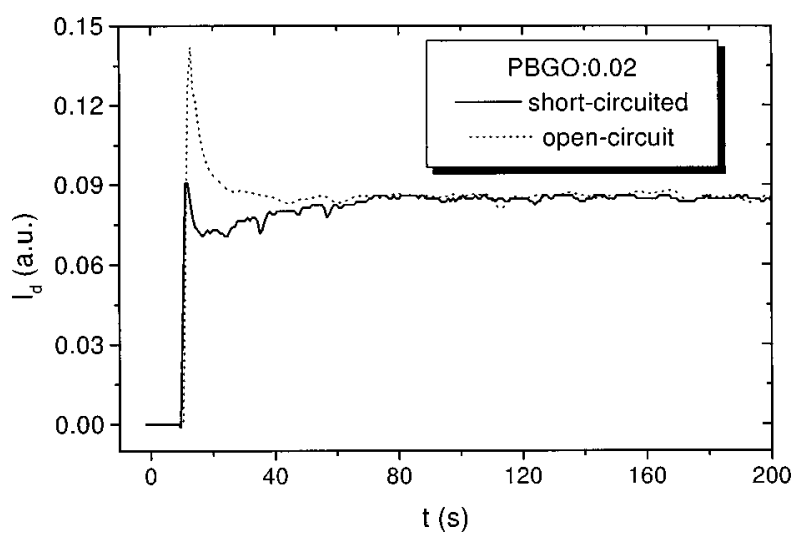

Fig. 4. Evolution of diffracted beam intensity $I_{d}$ for shortcircuited and open-circuited holographic recording in sample PBGO:0.02. The intensity of the writing beams is $I_{0}$ $=8 \mathrm{~W} / \mathrm{cm}^{2}$.

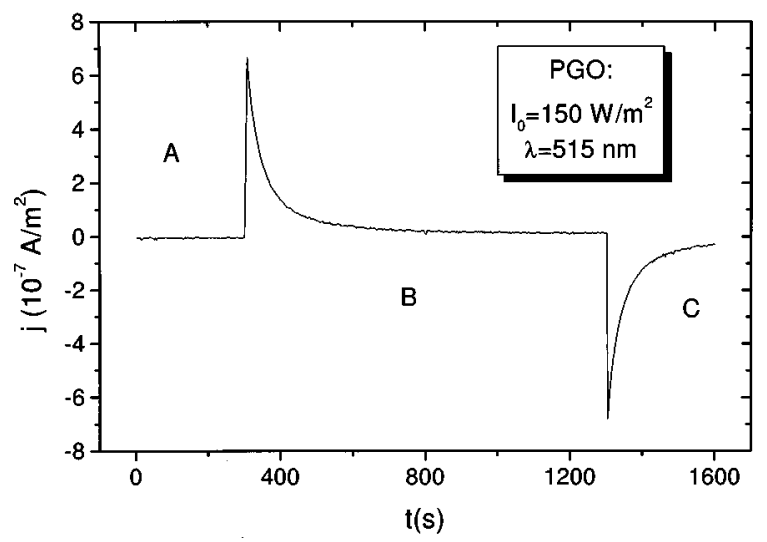

Fig. 5. Evolution of the pyroelectric current density $j$ before (area A of Fig. 1), during (area B), and after (area C) homogeneous illumination of sample PGO.

mum value (region C; see Fig. 5) can be described by an exponential law with a characteristic time constant of $\tau_{d}$ $=60 \mathrm{~s}$.

\section{DISCUSSION}

First we point out that the decrease in diffraction efficiency after the maximum is reached during holographic recording without preillumination cannot be attributed to the compensation of multiple gratings. From Fig. 1 we see that the diffracted beam intensity $I_{d}$ remains constant after the abrupt drop at the initial recording. The steady-state value is the same as that achieved by use of lower intensity. Several other factors may lead to a transient enhancement of diffraction efficiency during holographic recording in photorefractive crystals. Recently the same transient enhancement of photorefractive gratings in $\mathrm{Bi}_{4} \mathrm{Ti}_{3} \mathrm{O}_{12}$ was observed after preillumination of the sample with intense light. ${ }^{12}$ A light-induced variation of electron-hole competition is believed to be the origin of this effect. Although electron-hole competition was also found in our samples, ${ }^{10}$ here the enhancement of photorefractive gratings occurs without any preillumination. In addition, short-circuit recording decreases the degree of enhancement. There is no reason that shortcircuited crystal surfaces have any influence on electron- 
hole competition. Time-dependent fields such as a photovoltaic field can produce similar dynamic behavior during grating formation. ${ }^{13}$ But in our samples, as presented in Fig. 5, in the steady state the photocurrent is zero, indicating that photovoltaic effects are negligible. The absorption of light, however, can heat the sample and produce a pyroelectric field that may be compensated for by photoinduced charge carriers. In what follows, we consider this influence of pyroelectric fields during holographic recording in lead germanate crystals.

\section{A. Pyroelectric Fields}

For small grating spacings and with the use of cw laser light, thermal gratings produced by inhomogeneous illumination are small and can be neglected. ${ }^{3,14}$ However, photorefractive crystals can be heated by absorbing light and establish a strong pyroelectric field across the whole illuminated (heated) region. ${ }^{8}$ The pyroelectric field $E_{\text {pyro }}$ can be expressed by

$$
E_{\text {pyro }}=-\left(\partial P_{s} / \partial T\right)\left(\epsilon \epsilon_{0}\right)^{-1}\left(T-T_{0}\right),
$$

where $P_{s}$ is the spontaneous polarization, $\partial P_{s} / \partial T$ is the pyroelectric coefficient, $\epsilon$ is the dielectric constant, $\epsilon_{0}$ is the permittivity of vacuum, $T_{0}$ is the initial temperature of the sample (room temperature), and $T$ is the increased temperature after illumination. If the whole sample is illuminated, we consider only the heat convection between the crystal and the surrounding atmosphere. In addition, the anisotropic properties of the heat-conduction coefficient $h_{c}$ are not taken into account. Then the increase in crystal temperature is given as

$$
\frac{d T}{d t}=\left[P-h_{c} A\left(T-T_{0}\right)\right] / c_{p} m,
$$

where $P$ is the absorbed power, $m$ is the mass, and $c_{p}$ is the specific heat capacity. By combining it with the initial condition of $T=T_{0}$ at $t=0$, we can solve Eq. (2) to get the following result:

$$
T-T_{0}=k \tau_{H} I_{0}\left[1-\exp \left(-t / \tau_{H}\right)\right],
$$

where $k=[1-\exp (-\alpha d)]\left(c_{p} \rho d\right)^{-1}$ is the rate of temperature rise without considering heat conduction, $\tau_{H}$ $=c_{p} m / h_{c} A$ is the heat-relaxation time constant, $A$ is the area, $\rho$ is the density of the crystal, $\alpha$ is the absorption coefficient, and $d$ is the crystal thickness.

We now take sample PGO as an example to estimate the pyroelectric field. The pyroelectric coefficient is $\partial P_{s} / \partial T=-1.1 \times 10^{-4} \mathrm{C} \mathrm{K}^{-1} \mathrm{~m}^{-2} .^{15} \quad$ From the pyroelectric current measurement we can estimate the specific heat capacity $c_{p}$ by using the equation ${ }^{16}$

$$
j=\left(\partial P_{s} / \partial T\right) I_{0}[1-\exp (-\alpha d)] / c_{p} d \rho,
$$

which is valid for the first moment after the light is switched on. From Fig. 5 we obtain $c_{p}$ $=530 \mathrm{~J} \mathrm{~K}^{-1} \mathrm{~kg}^{-1}$. In addition, the parameter $\tau_{H}$ should be equal to $\tau_{d}$, which is defined as the relaxation time constant of the current after shutoff of the illumination in the pyroelectric current measurements. For sample PGO we thus assume that $\tau_{H}=60 \mathrm{~s}$. Other parameters are the sample's dimensions $2.40 \mathrm{~mm} \times 6.20 \mathrm{~mm}$ $\times 5.50 \mathrm{~mm}$, density $\rho=7.33 \times 10^{3} \mathrm{~kg} / \mathrm{m}^{3}$, and absorption coefficient $\alpha=3.2 \mathrm{~cm}^{-1}$ for $488 \mathrm{~nm}$. Consequently, for a beam intensity of $I_{0}=4 \mathrm{~W} / \mathrm{cm}^{2}$ and a time $t=1 \mathrm{~s}$ we get a temperature increase of $\Delta T=0.5 \mathrm{~K}$. Based on Eq. (1), a pyroelectric field $E_{\text {pyro }} \sim 1.8 \mathrm{kV} / \mathrm{cm}$ can be reached. Thus we conclude that the homogeneous pyroelectric field can be of great importance in holographic recording in lead germanate crystals.

\section{B. Compensation of the Pyroelectric Fields}

In contrast to experiments with an external electric field or a photovoltaic field, here a pyroelectric field will be compensated for by moving charges. ${ }^{8}$ This means that the pyroelectric field can act on the photorefractive grating only transiently, as we observed in our experiments.

The time for compensation of the pyroelectric field depends on the conductivity of the crystal, and thus the pyroelectric field decays exponentially with a time constant $\tau_{M}$ that is equal to the Maxwell time of the material. By combining Eqs. (1) and (3) and considering charge compensation, we can express the temporal evolution of the pyroelectric field as

$$
E_{\text {pyro }}(t)=\gamma \tau_{H} I_{0}\left[1-\exp \left(-t / \tau_{H}\right)\right] \exp \left(-t / \tau_{M}\right)
$$

where $\gamma=k\left(\partial P_{s} / \partial T\right)\left(\epsilon \epsilon_{0}\right)^{-1}$ is a constant, $\tau_{M}=\epsilon \epsilon_{0} / \sigma$ is the Maxwell time, and $\sigma$ is the conductivity. In our experiments dark conductivity is negligible compared with photoconductivity. In Fig. 6 we show values of the pyroelectric field as a function of time for different photoconductivities. It has to be mentioned that the parameters (see the caption to Fig. 6) that we have chosen here are average values obtained from measurements of different samples. From this figure we see that the pyroelectric field increases after the illumination beams are turned on, reaches a maximum value, and then decreases to zero. This process is similar to what we observe in the experiment to find the diffracted beam's intensity. The time $t_{m}$ that is necessary to reach the maximum pyroelectric field can be derived from Eq. (5) to be

$$
t_{m}=\tau_{H} \ln \left(1+\tau_{M} / \tau_{H}\right) .
$$

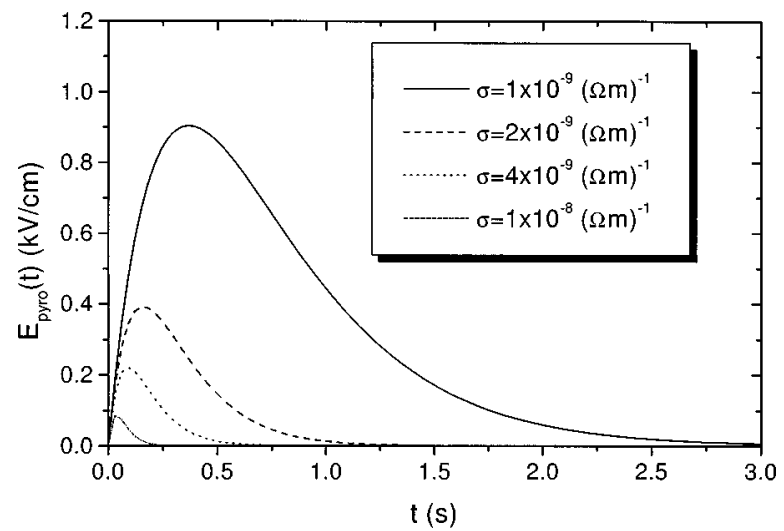

Fig. 6. Calculated evolution of pyroelectric field $E_{\text {pyro }}$ during homogeneous illumination of lead germanate crystals. The beam intensity is $I_{0}=4 \mathrm{~W} / \mathrm{cm}^{2}$, the thickness of the crystals is taken as $d=2.4 \mathrm{~mm}$, the absorption coefficient is $\alpha=2 \mathrm{~cm}^{-1}$, the pyroelectric coefficient is $\partial P_{s} / \partial T=-1.1 \times 10^{-4} \mathrm{C} \mathrm{K}^{-1} \mathrm{~m}^{-2}$, the heat capacity is $c_{p}=530 \mathrm{~J} \mathrm{~K}^{-1} \mathrm{~kg}^{-1}$, and the values of the photoconductivity are given in the inset. 


\section{Contribution of Pyroelectric Fields to Photorefractive Gratings}

In our experiments the grating spacing is kept at $1.2 \mu \mathrm{m}$; thus thermal gratings and gratings produced directly by modulation of the pyroelectric field can be neglected. The refractive-index modulation during holographic recording can be expressed as ${ }^{5}$

$$
E_{\mathrm{sc}}=-E_{\mathrm{pyro}}(t)-i E_{\text {diff }}, \quad E_{\text {diff }}=K k_{B} T_{0} / e,
$$

where $K$ is the amplitude of the grating vector, $k_{B}$ is the Boltzmann constant, and $e$ is the elementary charge of electrons. For small diffraction efficiencies $\eta(\eta \ll 1)$ we can write

$$
\eta=\left(\frac{\pi n^{3} r_{\mathrm{eff}} d}{2 \lambda \cos \theta}\right)^{2}\left(E_{\mathrm{pyro}}^{2}+E_{\text {diff }^{2}}^{2} .\right.
$$

A transient enhancement of diffraction efficiency, as presented in Fig. 1, is expected as a consequence of the transient increase in the pyroelectric field shown in Fig. 6 . Because of the large photoconductivity of the nominally pure sample, the characteristic time constant $\tau_{M}$ of the compensation charges is much smaller than those of PBGO:0.02 and PBGO:0.04. This is the reason why no large transient enhancement of photorefractive gratings is observed in the nominally pure sample. The same argument holds for the two PBGO samples: the conductivity of the sample with $4 \% \mathrm{Ba}$ is smaller than that with $2 \%$ $\mathrm{Ba}$, and the pyroelectric coefficient $\partial P_{s} / \partial T$ for the former is larger, too. Thus the enhancement factor is larger in PBGO:0.04 than in PBGO:0.02, although the absorption coefficient of the former is smaller. ${ }^{10}$

If the two $c$ faces are short-circuited and the whole sample is illuminated, no voltage is produced across the sample along the $c$ direction. As shown in Fig. 4, the enhancement factor $M$ in the short-circuited case becomes much smaller than that for open-circuit recording. The remaining weak enhancement may be due to the nonuniform illumination near both surfaces perpendicular to the $c$ axis. In these regions some charges are accumulated because of the smaller conductivity.

\section{Dependence on Light Intensity}

The intensity dependence of the transient enhancement factor $M$ shown in Fig. 2 can be explained with Eqs. (5), (6), and (8). The measured value $\tau_{H}$ of different samples is generally in the range of several tens of seconds, and the value of $\tau_{M}$ depends on intensity. For PBGO:0.02, $\tau_{M}$ can be expressed as $\tau_{M}=1.2 I_{0}{ }^{-0.6}$, where $I_{0}$ has to be in watts per square centimeter. Thus the ratio $\tau_{M} / \tau_{H}$ is generally much less than 1, and we can simplify Eq. (6) to $t_{m} \approx \tau_{M}$. Based on the definition of the transient enhancement factor $M$ we get the following expression:

$$
M=1+\left\{\xi \tau_{H} I_{0}\left[1-\exp \left(-1.2 I_{0}^{-0.6} / \tau_{H}\right)\right]\right\}^{2},
$$

where $\xi=(\gamma / 2.7) / E_{\text {diff }}$ is in units of square centimeters per joule. We use Eq. (9) to describe the experimental results presented in Fig. 2. The fit is shown as the solid curve, which shows good agreement with experiment (see also the discussion in Subsection 4.E).

The transient enhancement factor becomes much smaller when the grating is recorded with preillumination (Fig. 1). This result is expected because by preillu- mination the sample's temperature has already reached a steady-state value and the pyroelectric field has been compensated for by photoexcited charge carriers. From Eq. (9) we obtain that $M$ with preillumination should be only one quarter of the value without preillumination.

\section{E. Dependence on Dark Delay between Successive Recording Processes}

For recording with writing-beam intensity $I_{0}$, the amplitude of the temperature increase is saturated at $\Delta T_{0}$ $=\gamma I_{0} \tau_{H}$. In the dark the temperature decreases according to an exponential law with the time constant $\tau_{H}$. After a dark delay time $t_{d}$ we start a new recording cycle and assume that the initial internal field is approximately 0 because of the compensation. Then the temperature variation during the new recording is

$$
\Delta T=\Delta T_{0}\left[1-\exp \left(-t_{d} / \tau_{H}\right)\right]\left[1-\exp \left(-t / \tau_{H}\right)\right] .
$$

The corresponding transient enhancement factor is obtained as

$$
M=1+\left\{\xi I_{0}\left[1-\exp \left(-\tau_{M} / \tau_{H}\right)\right]\left[1-\exp \left(-t_{d} / \tau_{H}\right)\right]\right\}^{2},
$$

where $I_{0}=8 \mathrm{~W} / \mathrm{cm}^{2}$ and $t_{M}=0.34 \mathrm{~s}$. We use both Eqs. (9) and (11) to describe the experimental results presented in Figs. 2 and 3, applying the same set of parameters $\tau_{H}$ and $\xi$. The fits are shown in these two figures as solid curves and are in good agreement with the experimental values. The fitted parameters are $\tau_{H}=34 \mathrm{~s}$ and $\xi=0.3$. The time constant $\tau_{H}$ is of the same order as the value measured directly in other samples. The fit value of $\tau_{H}$ is smaller than that obtained from pyroelectric current measurements, which may be due to that fact that in holographic measurements the whole crystal is not homogeneously illuminated. Heat conductivity also leads to additional heat losses. In principle, $\xi$ can be calculated directly if the pyroelectric coefficient $\partial P_{S} / \partial T$, the specific heat capacity $c_{p}$, and the diffusion field $E_{\text {diff }}$ are known. Although the first two of these parameters have not been determined experimentally for PBGO:0.02, we can estimate $\xi$ by assuming that these two parameters are approximately the same as those for PGO. Then the calculated value is $\xi=0.3$, which is equal to the value that we obtained from the fit of Eqs. (9) and (11) to the experimental results. This does not mean that the theory perfectly describes the phenomenon, because we have made some assumptions and some parameters are not known exactly. But we can clearly use the theory presented for an explanation of the influence of pyroelectric fields in our samples during holographic recording.

In conclusion, we have reported a transient enhancement of photorefractive gratings in $\mathrm{Pb}_{5} \mathrm{Ge}_{3} \mathrm{O}_{11}$ and $\left(\mathrm{Pb}_{1-x} \mathrm{Ba}_{x}\right)_{5} \mathrm{Ge}_{3} \mathrm{O}_{11}$. The dynamic behavior of grating formation and the dependence of the transient enhancement of photorefractive gratings on writing-beam intensity and dark delay between two successive recording processes can be described by a homogeneous pyroelectric field because of light absorption. We pointed out that both the pyroelectric field and photoconductivity are responsible for the transient enhancement at the initial stage of holographic recording. All experiments were 
carried out with cw laser light, which means that the homogeneous pyroelectric field is strong in this material. But, in crystals with large photoconductivity, pyroelectric fields are always compensated for by charge accumulation at the boundary between dark and illuminated regions. Thus the pyroelectric effect can be controlled both by experimental conditions such as intensity and preillumination and by tailoring of material parameters such as doping and thermal treatments. Finally, we mention that further experiments using Fe-doped crystals with larger absorption show even more significant transient enhancement $\left(M>7\right.$ for $\left.I_{0}=16 \mathrm{~W} / \mathrm{cm}^{2}\right)$ by homogeneous pyroelectric fields. Indeed, there is a demand for more knowledge of the homogeneous pyroelectric fields during holographic recording in photorefractive materials. Applications of this effect need further consideration, too.

\section{ACKNOWLEDGMENTS}

Financial support by the Deutsche Forschungsgemeinschaft (SFB 225, A1, A6) and the Friedrich-Ebert-Stiftung are gratefully acknowledged.

Present address: Department of Electrical Engineering, California Institute of Technology, Mail Stop 136-93, Pasadena, California 91125.

\section{REFERENCES}

1. P. Günter and J.-P. Huignard, Photorefractive Materials and Their Applications (Springer-Verlag, Berlin, 1988), Vols. I and II.

2. N. V. Kukhtarev, V. B. Markov, S. Odoulov, M. S. Soskin, and V. L. Vinetskii, "Holographic storage in electrooptic crystals. I. Steady state," Ferroelectrics 22, 949 (1979).

3. V. L. Vinetskii and M. A. Itskovskii, "Pyroelectric mechanism of the holographic recording," Ferroelectrics 18, 81 (1978).
4. S. Ducharme, "Pyroelectro-optic phase gratings," Opt. Lett. 16, 1791 (1991).

5. K. Buse, "Thermal gratings and pyroelectrically produced charge redistribution in $\mathrm{BaTiO}_{3}$ and $\mathrm{KNbO}_{3}$," J. Opt. Soc. Am. B 10, 1266 (1993).

6. W. W. Clark III, G. L. Wood, M. J. Miller, E. J. Sharp, G. J. Salamo, B. Monson, and R. R. Neurgaonkar, "Enhanced photorefractive beam fanning due to internal and external electric fields," Appl. Opt. 29, 1249 (1990).

7. P. L. Ramazza and M. Zhao, "Experimental study of twowave mixing amplification in Cu-doped KNSBN," Opt. Commun. 102, 93 (1993).

8. N. Korneev, D. Mayorga, S. Stepanov, A. Gerwens, K. Buse, and E. Krätzig, "Enhancement of photorefractive effect by homogeneous pyroelectric fields," Appl. Phys. B: Lasers Opt. 66, 393 (1998).

9. W. Królikowski, M. Cronin-Golomb, and B. S. Chen, "Photorefractive effect in ferroelectric lead germanate," Appl. Phys. Lett. 57, 7 (1990).

10. X. Yue, S. Mendricks, Y. Hu, H. Hesse, and D. Kip, "Photorefractive effect in doped $\mathrm{Pb}_{5} \mathrm{Ge}_{3} \mathrm{O}_{11}$ and in $\left(\mathrm{Pb}_{1-x} \mathrm{Ba}_{x}\right)_{5} \mathrm{Ge}_{3} \mathrm{O}_{11}$," J. Appl. Phys. 83, 3473 (1998).

11. M. C. Bashaw and J. F. Heanue, "Quasi-stabilized ionic gratings in photorefractive media for multiplex holography," J. Opt. Soc. Am. B 14, 2024 (1997).

12. X. Yue, K. Buse, F. Mersch, E. Krätzig, and R. A. Rupp, "Diffraction efficiency enhancement of photorefractive gratings in $\mathrm{Bi}_{4} \mathrm{Ti}_{3} \mathrm{O}_{12}$ at low temperatures," J. Opt. Soc. Am. B 15, 142 (1998).

13. C. Gu, J. Hong, H. Y. Li, D. Psaltis, and P. Yeh, "Dynamics of grating formation in photovoltaic media," J. Appl. Phys. 69, 1167 (1991).

14. K. Buse and K. H. Ringhofer, "Pyroelectric drive for lightinduced charge transport in the photorefractive process," Appl. Phys. A: Mater. Sci. Process. 57, 161 (1993).

15. K.-H. Hellwig, ed., Landolt-Börnstein-Numerical Dato and Functional Relationships in Science and Technology, New Series (Springer-Verlag, Berlin, New York, 1981), Vol. III/16.

16. K. Buse, U. Van Stevendaal, R. Pankrath, and E. Krätzig, "Light-induced charge transport properties of $\mathrm{Sr}_{0.61} \mathrm{Ba}_{0.39} \mathrm{Nb}_{2} \mathrm{O}_{5}$ :Ce crystals," J. Opt. Soc. Am. B 13, 1461 (1996). 